\title{
Use of an Artificial Neural Network to Assess the Degree of Training of an Operator of Selected Devices Used in Precision Agriculture
}

\author{
Karolina Trzyniec ${ }^{1, *}$ and Adam Kowalewski ${ }^{2}$ \\ 1 Ergonomics and Production Processes, Department of Machinery Operation, Faculty of Production and \\ Power Engineering, University of Agriculture, 30-149 Cracow, Poland \\ 2 Department of Automatic Control and Robotics, Faculty of Electrical Engineering, Automatics, \\ Computer Science and Biomedical Engineering, AGH University of Science and Technology, 30-059 Cracow, \\ Poland; ako@agh.edu.pl \\ * Correspondence: karolina.trzyniec@urk.edu.pl
}

Received: 30 October 2020; Accepted: 26 November 2020; Published: 30 November 2020

\begin{abstract}
The article concerns the issue of automatic recognition of the moment of achieving the desired degree of training of an operator of devices used in precision agriculture. The aim of the research was to build a neural model that recognizes when an operator has acquired the skill of operating modern navigation on parallel strips used in precision agriculture. To conduct the test, a standard device to assist the operator in guiding the machine along given paths, eliminating overlaps, was selected. The thesis was proven that the moment of operator training (meaning driving along designated paths with an accuracy of up to eight centimeters) can be automatically recognized by a properly selected artificial neural network. This network was learned on the basis of data collected during the observation of the operator training process, using a criterion defined by experts. The data collected in the form of photos of the actual and designated route was converted into numerical data and entered into the network input. The output shows the binary evaluation of the trip. It has been shown that the developed neural model will allow the determining of the moment when operators acquire the skills to drive a vehicle along the indicated path and thus shorten the training time.
\end{abstract}

Keywords: GPS; artificial neural network; navigation; precision agriculture; operator training

\section{Introduction}

The development of agricultural mechanization meant that the work of a modern operator is less and less dependent on the direct operation of an agricultural machine [1] (pp. 138-139). The operator's main task is usually to manage processes by efficiently operating automated and computerized signaling and control devices [2] (pp. 12-15). Monitoring the work of machines with the use of appropriate signaling and control devices cooperating with those machines is one of the opportunities of using modern, precision agriculture systems. These systems not only enable the positioning of a tractor or agricultural machine in the field, but, above all, support the operator's work by accurately guiding the vehicle along a designated path [3] (pp. 120-122). However, to keep the vehicle in the right course in the field, the operator must acquire the skills to properly read the navigation panel indications and to drive the vehicle according to those indications. From the navigation panel, the operator can read the current vehicle position and direction of travel in relation to the entire recorded area. A practical difficulty that can be encountered by tractor operators cooperating with navigation systems on parallel strips is the problem of maintaining a straight drive line, without deviations greater than 20-30 cm, especially when driving on a field with uneven borders or situated on a hill [3] (pp. 120-122). The pace of acquiring skills for faultless driving in a designated track is different for each operator. 
Observation of the field from the tractor cabin does not allow for reliable assessment of test drives (performed during the operator training), and the small size of the navigation screen prevents accurate analysis of the entire trajectory. For this reason, it is difficult to determine when the operator will acquire relevant skills. On the other hand, objective determination of this moment also allows for deciding when the training may be interrupted. Early termination of the training (once it is certain that the operator already has the required skills) can lead to significant savings in time and material resources (e.g., fuel needed to perform training drives).

A lot of scientific research in the field of artificial intelligence focuses on the implementation of IT tools for autonomous driving of vehicles, including agricultural or forestry tractors, after crops. Autonomous driving of agricultural machinery using information from the Global Navigation Satellite System (GNSS) is an issue developed in many countries around the world [4]. Machine constructors, in cooperation with IT specialists, create unmanned ground vehicles, which are a perfect solution for modern, intelligent farms. Examples of such solutions are described by Gonzales de Santos et al. [5], Chemhengcharoen et al. [6], Hasníková et al. [7] or Petterson [8]. Autonomous vehicles guarantee optimal use of the field surface and perfectly even passages. However, they are still too expensive for most farmers. It therefore seems that good training of the navigation operator on parallel lanes will provide a similar effect at lower costs. Automatic path tracking keeps you in control of the ride. Samuel et al. [9] wrote about the use of artificial intelligence to control an autonomous vehicle using path tracking, and Ashraf et al. [10] used neural networks for vehicle navigation on sloping terrain. So far, however, no artificial intelligence tools have been used to support real operator training.

\section{Purpose and Scope of the Research}

The objective of the research was to build a neural model that recognizes when an operator acquired the skill of operating modern navigation on parallel strips used in precision agriculture. The neural model that recognizes the moment of operator training will determine the speed (pace) of acquiring professional skills. Having a tool that allows monitor of the progress of operator training will reduce training time.

Based on the presented research objective, the following hypothesis was formulated: the moment of achieving the desired degree of operator training can be automatically recognized by a properly selected artificial neural network, learned on the basis of data collected during observation of the training process of other operators, using a criterion specified by experts.

To prove the hypothesis, the following stages of research were required:

- conducting training for 15 potential operators of a vehicle cooperating with navigation on parallel strips by a person supervising the training;

- collecting data characterizing the trajectory of the vehicle controlled by the operator during the training;

- assessment of operator skills by the person supervising the researches, according to accepted and justified criteria;

- developing a collection that teaches an artificial neural network based on the results of experimental research conducted;

- determining the structure of the neural model and its teaching;

- analysis of results of teaching of developed neural networks.

The term "potential operators" was used above. This concept was used by the authors to explain that the aim of the experiment was not to professionally prepare farmers, but to determine the speed of learning how to operate the Local Positioning System (LPS, a technology used to obtain information about the position or location of objects in relation to a local field or area). In fact, acquiring these skills means that each operator participating in the training is no longer "potential", but is an operator in the full sense of the word. This means that it can drive the vehicle along the paths assigned to it with the appropriate, set accuracy. 


\section{Research Methodology}

\subsection{Data}

In order to check the level of difficulty in operating the signaling and control devices equipped with the LPS (Local Positioning System), training was provided for fifteen potential operators of the vehicle cooperating with the Trimble navigation panel. The Trimble CFX 750 model was used for the tests because it was the equipment of the Laboratory of Precision Agriculture of the University of Agriculture. Due to the high operating costs of the agricultural tractor, a passenger car equipped with a fully functional steering system was used for field tests. The experiments included training for six women and nine men aged 19-58. The average age was 34 years. Table 1 presents the general characteristics of the group of people.

Table 1. Characteristics of people participating in field experiments.

\begin{tabular}{ccccc}
\hline $\begin{array}{c}\text { Designation of the Person } \\
\text { Participating in the Experiment }\end{array}$ & $\begin{array}{c}\text { Gender of the Person } \\
\text { (F-Female, M-Male) }\end{array}$ & $\begin{array}{c}\text { Age } \\
\text { (Years) }\end{array}$ & $\begin{array}{c}\text { Work Experience * } \\
\text { (Years) }\end{array}$ & $\begin{array}{c}\text { Knowledge of the LPS System } \\
\text { (According to the Adopted Scale }\end{array}$ \\
\hline A & F & 29 & 4 & + \\
B & F & 29 & 11 & + \\
C & F & 23 & 2 & + \\
D & F & 29 & 1 & + \\
E & F & 53 & 35 & + \\
F & F & 19 & 1 & + \\
G & M & 34 & 17 & ++ \\
H & M & 53 & 36 & + \\
I & 29 & 11 & ++ \\
J & M & 24 & 6 & + \\
L & M & 30 & 9 & + \\
M & M & 31 & 4 & ++ \\
N & M & 58 & 41 & + \\
O & M & 36 & 18 & + \\
\hline
\end{tabular}

${ }^{*}$ Work experience is understood as the time of having a driving license. ${ }^{* *},+-$ theoretical knowledge of the LPS (Local Positioning System), ++-theoretical and practical knowledge of the LPS. Practical knowledge of the LPS system means that the person participating in the experiment operated the navigation panel on parallel strips at least once in their life.

The training of potential device operators was conducted by a real trainer (fully trained device operator) who followed and recorded the results of the training during few dozen test drives. The number of drives averaged 46 and was partially selected arbitrarily, according to the subjective opinion of the supervisor, i.e., the trainer. Based on the observation of the course of drives, the trainer decided to stop training at a moment when during a minimum of three consecutive drives the trajectory was as close as possible to the preset trajectory, because it turned out that, with a $100 \%$ probability, the operator who drove correctly three times is able to do it correctly again. In practice, this meant that the path made by the operator coincided with the set path (parallel to the previously made). The arbitrariness of the decision was based on the fact that the result of the trainer's observation decided to end the training of a given operator (or further continuation of the training).

The number of correctly made drives depended on the operator's level of training. The better the mastery of the technique (method) of driving, the greater the probability of keeping the established driving route; and vice versa: the less trained the operator, the more difficult it is to maintain the preset route of drive.

The arbitrariness of the decision on three subsequent, correctly made drives as a criterion for sufficient operator training, resulted from observations conducted by the trainer. The idea of assessing the correctness of driving a preset track with a vehicle by a learning operator is presented in Figure 1. 


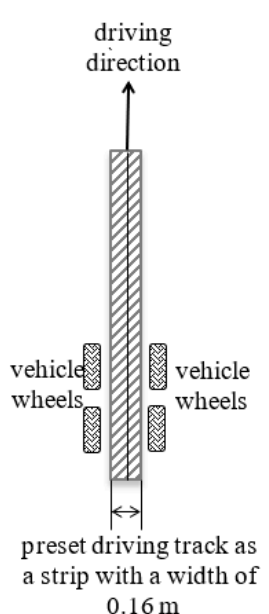

a

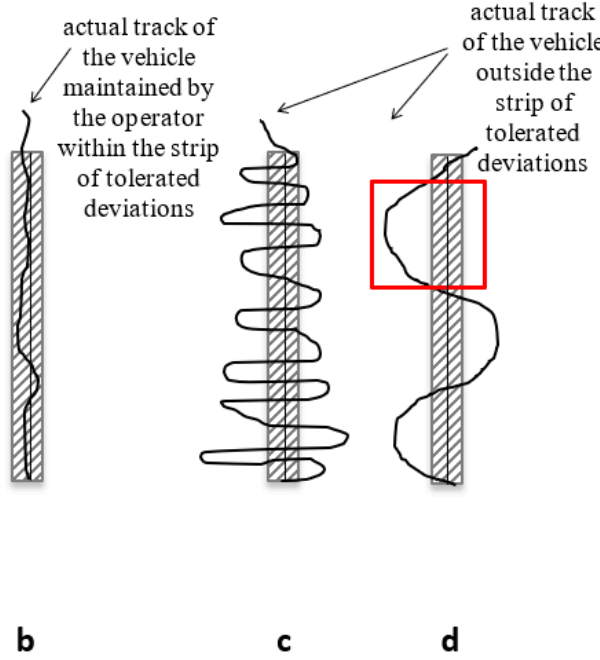

Coordinates of points

A, A', B, B':

$A\left(x_{A}, y_{A}\right)$

$\mathrm{A}^{\prime}\left(\mathrm{x}_{\mathrm{A}}, \mathrm{y}_{\mathrm{A}^{\prime}}\right)$

$\mathrm{B}\left(\mathrm{x}_{\mathrm{B}}, \mathrm{y}_{\mathrm{B}}\right)$

$\mathrm{B}^{\prime}\left(\mathrm{x}_{\mathrm{B}}, \mathrm{y}_{\mathrm{B}^{\prime}}\right)$

Figure 1. Horizontal plan showing the preset driving track and the actual driving track; (a)—tractor driving direction on a $20 \mathrm{~m}$ track, tolerance deviation strip $0.16 \mathrm{~m},(\mathbf{b})$ —correct drive (driving track maintained in the tolerated deviations track), (c,d)-incorrect drives; despite the differences, classified the same, i.e., as an incorrect drive, (e) - spatial parameters of deviations of the actual drive track from the preset track.

The course of field experiments was as follows:

1. The operator was tasked with driving the preset track by the trainer explaining how to keep the vehicle on the designated section.

2. The operator started the engine: after switching on the gear, driving at a speed of $6-7 \mathrm{~km} / \mathrm{h}$, the operator drove the preset distance (observing the vehicle position on the GPS screen),

3. The driving parameters, i.e., the current position of the vehicle, were recorded in the form of photographs (Figure 2).

4. Recorded momentary vehicle positions were formatted for further (computer) analysis using a graphic-numerical conversion program.

5. Converted numerical data (in the $x$ and y coordinate system, as in Figure 3 ) formed a database characterizing the given drive.

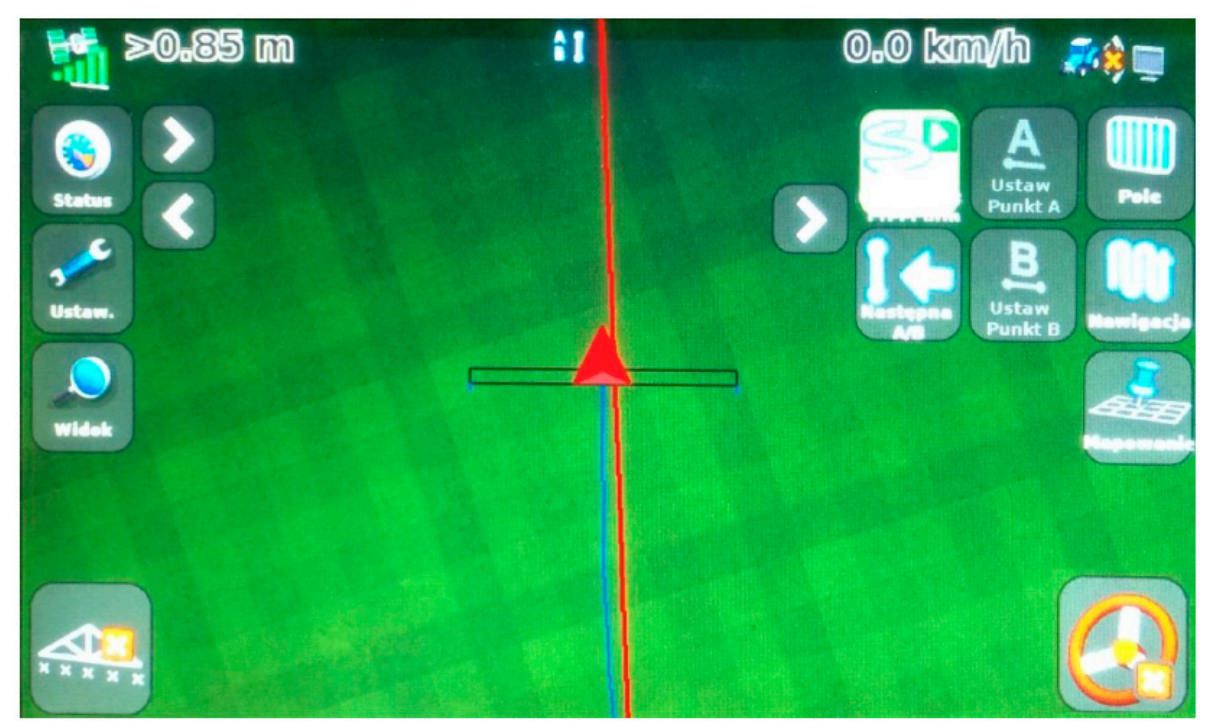

Figure 2. Image seen by the operator on the screen of the Trimble CFX 750 navigation panel—sample recording of the preset (red line) and actual (blue line) vehicle track. 


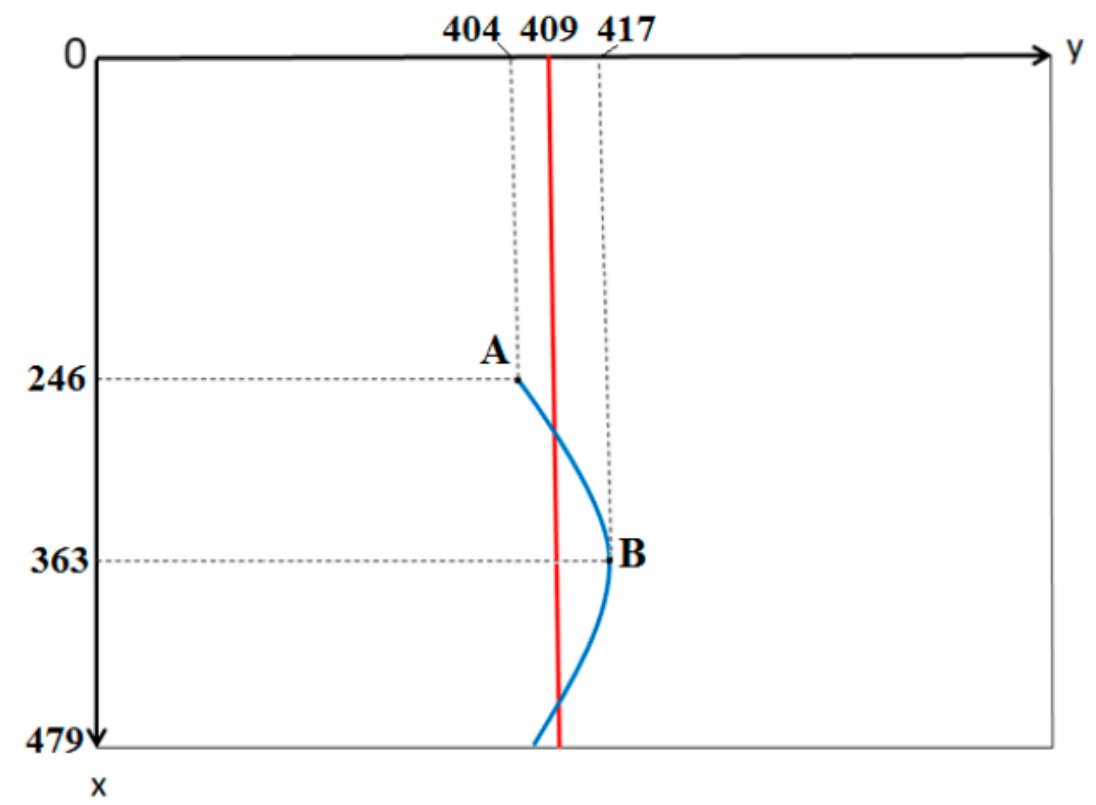

Figure 3. Determined vehicle position at points $A$ and $B$, in the $x$ and $y$ system, expressed by the number of pixels on the screen (after conversion to numerical data).

Figure 2 shows the discrepancy between the path given to the operator and the actual path. After each (incorrect) drive, the operator received recommendations from the instructor to better control straightness of the drive and use of navigation indications. Navigation indications mean not only the displayed, instantaneous vehicle positions relative to the preset track, but also three-color signaling electronic elements (diodes) located in the upper part of the navigation panel housing. The correct position of the vehicle relative to the designated track (i.e., location in the strip of tolerated deviations $0.16 \mathrm{~m}$ wide) is signaled by three green diodes. Each tilting of the vehicle axis to the right, outside the tolerance range is signaled by red diodes on the left and vice versa: any tilting of the vehicle axis to the left is signaled by red diodes on the right. The total number of indicator diodes is 27 (three green and 24 red ones). The list of available options of the navigation panel settings (i.e., menu) allows to manually set the width of the tolerated deviations strip and the width of the deviation (called in the menu as the stroke value) from this strip corresponding to one red diode.

A stroke value of $0.08 \mathrm{~m}$ was programmed in the experiment because it is the width corresponding to one pixel in the image.

The collected photos, on which both routes overlap, have been ordered in the order of drives and cataloged separately for each operator.

\subsection{Data Conversion}

The collected images of the training process (i.e., individual drives, on the basis of which conclusions were drawn about the current skills of the operator) were converted into numerical tables. Coordinates $\left\langle\mathrm{x}_{\mathrm{iR}}, \mathrm{y}_{\mathrm{iR}}>\right.$ of individual points of the actual trajectory of the machine and $\left\langle\mathrm{x}_{\mathrm{iZ}}, \mathrm{y}_{\mathrm{iZ}}\right\rangle$ of the preset (determined) trajectory were recorded in those tables (two for each photographic registration of the drive). The number of pixels recorded on each preset trajectory was $p=479$. The number of pixels recorded on each real trajectory was $p^{\prime}=234$. Since the actual route section was always $20 \mathrm{~m}$, it was calculated that one pixel in the image corresponds to a distance of $0.08 \mathrm{~m}$. Entering all values of the vectors $\langle x\rangle$ and $\langle y\rangle$, which are a digital representation of both routes, would cause the size of the network to be too large (caused by too many input vectors). The issue of input data representation was solved by determining the differences of the real trajectory from the preset trajectory. Because the $x$ vectors of both routes have the same position $\left(x_{i R}=x_{i z}\right)$, and the trajectories: the real and the preset ones differ in the position of the y vectors, the sets of the blue and red strips coordinates have 
been put together, and the absolute value of the difference of vectors: $y_{i z}$ and $y_{i R}$ was calculated. Calculations were made for all drives of each operator.

During the drive, the operator was maintaining or was not maintaining the vehicle on a preset track. The track was $20 \mathrm{~m}$ long (234 pixels on the recorder screen). Each position of the vehicle along the track ( $\mathrm{x}$ axis) corresponds to the difference in position of the vehicle relative to a preset track, expressed on the $y$ axis. Pairs of numbers $x_{n} y_{n}$ correspond to the momentary position of the vehicle.

The variability of differences in deviations from a preset track was expressed by the function $y=f(x)$ as a polynomial. The physical interpretation of a polynomial is as follows: if the momentary position of the vehicle on the track is known ( $\mathrm{x}$ axis), then the polynomial allows to determine the deviation from the track. The polynomial, obtained (by approximation) based on data from one drive, had the following form: $y=a_{n-1} x^{n-1}+a_{n} x^{n}+\ldots+a_{1} x^{1}+a_{2} x^{0}$.

Thanks to the introduction of polynomial approximation at the input of the neural network assessing the correctness of the drive, several (three or four) polynomial coefficients (real numbers multiplied by the powers of $\mathrm{x}$ ) describing the errors made during this drive could be introduced instead of 234 coordinate pairs describing the preset and actual vehicle trajectory. Only thanks to this task was it possible to effectively use a neural network of reasonable sizes (in the sense of the number of input elements and, consequently, the number of weighting factors necessary to be determined during the teaching process).

Polynomials were determined using the function $\mathrm{p}=$ polyfit() in the Matlab program. The approximation polynomial degree $\mathrm{N}=3$ was determined. At $\mathrm{N}>3$ all coefficients a ix for values $\mathrm{i}>3$ were zero. However, with the degree of polynomial $\mathrm{N}=3$, most coefficients at the highest power were still zero, therefore the following two values of the approximation polynomial degree were considered: $\mathrm{N}=2$ and $\mathrm{N}=3$.

All entered data—separately for each drive—formulated second and third degree polynomials. The usefulness of second and third degree polynomials for teaching the neural networks was evaluated experimentally by introducing the coefficients of those polynomials into the calculations. Experiments with neural networks have shown that both polynomials are useful in this case, although the network using the second degree polynomial coefficients achieved greater efficiency (i.e., learned better).

Below are examples of coefficients of polynomials with the degree $\mathrm{N}=2$ and $\mathrm{N}=3$ describing the drives considered to be correct (Table 2) and the drives with too large deviation from the preset trajectory (Table 3).

Table 2. Polynomial coefficients with the degree $\mathrm{N}=2$ and $\mathrm{N}=3$ for selected correct drives (i.e., drives maintained in the designated driving track).

\begin{tabular}{ccccccc}
\hline \multicolumn{3}{c}{$\begin{array}{c}\text { Polynomial Coefficients } \\
\text { with a Degree } \mathbf{N}=\mathbf{2}\end{array}$} & \multicolumn{5}{c}{$\begin{array}{c}\text { Polynomial Coefficients } \\
\text { with a Degree } \mathbf{N}=\mathbf{3}\end{array}$} \\
\hline $\mathrm{a} 2$ & $\mathrm{a} 1$ & $\mathrm{a} 0$ & $\mathrm{a} 3$ & $\mathrm{a} 2$ & $\mathrm{a} 1$ & $\mathrm{a} 0$ \\
\hline 0.0000 & -0.0015 & 1.1931 & 0.0000 & -0.0001 & 0.0254 & -1.9389 \\
0.0000 & 0.0000 & 1.0000 & -0.0000 & 0.0000 & -0.0000 & 1.0000 \\
0.0000 & -0.0011 & 1.1821 & -0.0000 & 0.0000 & -0.0053 & 1.6847 \\
\hline
\end{tabular}

Table 3. Polynomial coefficients with the degree $\mathrm{N}=2$ and $\mathrm{N}=3$ for selected incorrect drives (i.e., drives outside the designated driving track).

\begin{tabular}{ccccccc}
\hline \multicolumn{2}{c}{$\begin{array}{c}\text { Polynomial Coefficients } \\
\text { with a Degree } \mathbf{N}=\mathbf{2}\end{array}$} & \multicolumn{4}{c}{$\begin{array}{c}\text { Polynomial Coefficients } \\
\text { with a Degree } \mathbf{N}=\mathbf{3}\end{array}$} \\
\hline $\mathrm{a} 2$ & $\mathrm{a} 1$ & $\mathrm{a} 0$ & $\mathrm{a} 3$ & $\mathrm{a} 2$ & $\mathrm{a} 1$ & $\mathrm{a} 0$ \\
\hline 0.0002 & -0.1368 & 28.4187 & 0.0000 & -0.0001 & -0.0501 & 18.2690 \\
0.0001 & -0.0432 & 8.3167 & 0.0000 & -0.0001 & 0.0076 & 2.4524 \\
0.0000 & -0.0289 & 5.7749 & 0.0000 & -0.0006 & 0.2091 & -22.1769 \\
\hline
\end{tabular}


In fact, the coefficients of polynomials, in the whole range of their variability, express various variants of the operator's training level (acquisition of driving skills).

In theory, it is possible that the coefficients are the same for the same operator-or different operators. This would mean that (practically), the method of driving by the operator (operators) is identical (exactly reproducible method). The vehicle along the entire route (measuring section) would follow exactly the same track (each $x$ value would have the same $y$ value).

However, in practice the polynomial coefficients are different for each drive (and for each operator). This means that each of the operators drove the vehicle differently. Although driving was different, it was possible to determine-according to the adopted criterion, i.e., the correct three drives-at which time the driver has already acquired the appropriate skills. Different cases (made paths), formally described with polynomials with different coefficients $a_{n}, a_{n-1}, \ldots, a_{1}, a_{0}$, teach the neural network to recognize the moment of acquiring driving skills.

The method of presenting input data in the form of polynomial coefficients approximating the trajectory error was developed on the basis of research work conducted by the Szymczyk [11] (pp. 955-960), in which the problem of assessing the usefulness of neural networks for analysis, processing and interpretation of GPR signals was considered. The authors' review of the literature shows that the method presented in the article has not yet been used to solve the problem of training operators of equipment used in precision agriculture.

\subsection{Application of Polynomial Coefficients for Neural Network Learning}

Polynomial coefficients in the three-way system, i.e., polynomial coefficients of three successive drives were introduced to teach the neural network. For example: the first group were drives numbered 1,2 and 3, the second group were drives 2,3 and 4, the third group were drives 3,4 and 5, etc. The idea was to make a decision about a satisfactory level of operator training based on observations of repetitive correct drives. Incidental (accidental) single correct drives occurring in insufficiently trained operators did not lead to wrong decisions.

A binary assessment of three consecutive drives was introduced to the output of the neural network. This rating was only 1 if all three consecutive drives were correct. By giving each drive a score of $0-1$, the neural network was learned to detect this stage of training (in the literal sense: those three successive drives) when the operator was already trained (has correctly driven on the designated section).

A general, simplified diagram of the neural networks used is shown in Figures 4 and 5.

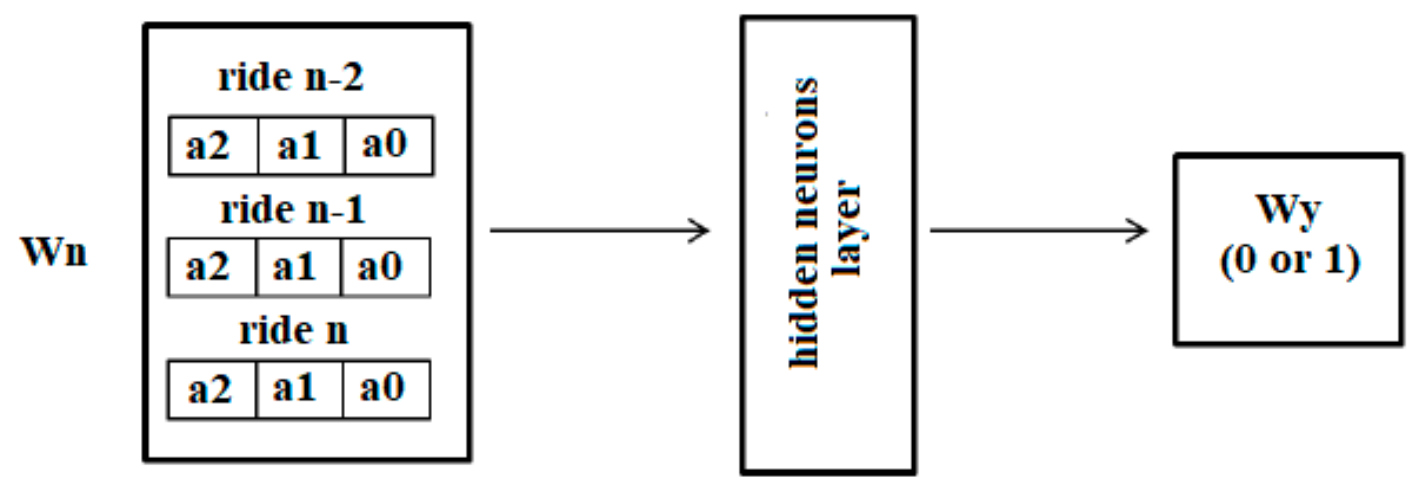

Figure 4. Diagram of a neural network using second degree polynomial coefficients for learning. a2, a1, a0-polynomial coefficients characterizing specific drives, $\mathrm{Wy}$-operator assessment result: 0 -not trained, 1 -trained. 


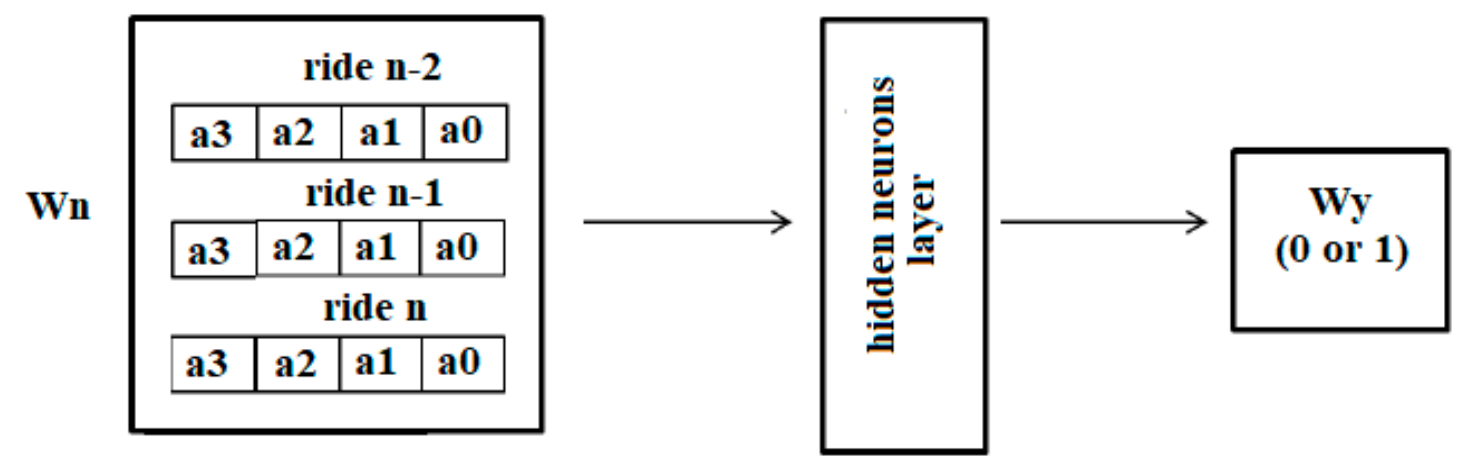

Figure 5. Diagram of a neural network using third degree polynomial coefficients for learning. a3, a2, a1, a0—-polynomial coefficients characterizing specific drives, $\mathrm{Wy}$-operator rating result: 0—not trained, 1-trained.

The prepared teaching set included 657 drive packages for each of the polynomial stages considered together with the assessment of each three drives. Assessments determining the operator's level of training ranged from $0-1$. Therefore, the problem analyzed in the work was based on the classification into two groups: a group of correct drives maintained in a preset track (assessed as 1) and a group of drives with too large deviation from the preset track (assessed as 0 ). In the analyzed problem, the elements (objects) in each group are represented in the same way-as polynomial coefficients of the same degree, but each object differs from other objects by the values of the coefficients. Taking into account the analysis of the literature on the subject [11] (pp. 955-960) [12-17], it was found that for the problem presented in the paper the most useful would be: multilayer perceptron network-MLP (Multilayer Perceptron) and a network with radial base functions-RBF (Radial Basis Function).

MLP networks are widely used in many areas because they are universal approximators and excellent pattern classifiers [18]. On the other hand, neural networks with radial base functions (RBFs) are also used in solving classification problems, because they are distinguished by properties that allow better mapping of the local characteristics of the modeled process. The purpose of classifying network training is to model a data generator to provide the best possible prediction when input data is known [19]. The problem of selecting the number of hidden layer neurons, which largely determines the effectiveness of the network (i.e., the degree of mapping accuracy) and the ability to generalize the network, was solved by using the Automatic Network Designer module that is part of the Statistica Neural Networks StatSoft package.

The experiment analyzed neural networks of various architectures, including those with many hidden layers. The use of many hidden values may result in the network overfitting, consisting in the network's over-adaptation only to the training data. To avoid this problem, the data set has been divided into: training data, test data and validation data. In addition, a series of experiments were carried out iteratively with each network configuration and the best configurations (that is, those with the lowest validation error values) were kept.

\section{Analysis of Modeling Results}

The neural model of operator's cooperation with the vehicle driven according to the indications of navigation on parallel strips was subjected to learning. To conduct the simulation, a database was developed containing 657 test drive packages with their binary assessment. The designed network using the second degree polynomial coefficients consisted of nine neurons for each input layer vector and one neuron in the output layer. For the network learned based on the third degree polynomial coefficients, the number of neurons in the input layer vector was 12 . Those numbers ( 9 and 12) result from the number of coefficients of polynomials $\mathrm{N}=2$ and $\mathrm{N}=3$ degree describing three consecutive drives. One thousand MLP networks using $N=2$ polynomial coefficients and 1000 MLP networks 
using $\mathrm{N}=3$ polynomial coefficients were created. The minimum number of neurons in the hidden layer was 1 and the maximum number of neurons in the hidden layer was 25.

The best results were achieved by the MLP 9-22-2 network. Therefore, it is a MLP network with nine input neurons, 22 hidden layer neurons and two output neurons. This network has achieved learning quality of over $99 \%$, using the BFGS learning algorithm. The first layer of neurons (i.e., the input layer) has a permanently hidden activation function (here: exponential function). The second layer (i.e., the hidden neuron layer) uses the so-called output activation function to process the entered data. The most commonly used functions include: linear, logistic, softmax ones. To solve the problem considered in the paper, the softmax function was selected (automatically, in the Statistica Neural Networks StatSoft package)—the network with the best parameters (i.e., with the highest efficiency) used this activation function.

After analyzing various structures of the neural network using the polynomial coefficients with a degree of $\mathrm{N}=3$, it was empirically confirmed that the best learning results are achieved by a network of 16 neurons in a hidden layer (MLP network 12-16-2). The selected network achieves learning quality of over $98 \%$, using the BFGS learning algorithm and the output softmax activation function. The quality of this network is comparatively as high as the quality of the MLP network using second degree coefficients (the difference is only approx. $0.23 \%$ ).

After testing the MLP network learning results, 1000 RBF networks using $\mathrm{N}=2$ polynomial coefficients and $1000 \mathrm{RBF}$ networks using $\mathrm{N}=3$ polynomial coefficients were created. The minimum number of neurons in the hidden layer was 1 and the maximum number of neurons in the hidden layer was 25. The analysis of the tested network structures showed that the RBF network using the polynomial coefficients with the degree $\mathrm{N}=2$ achieves the best learning results for 2 and 15 neurons in the hidden layer (RBF 9-2-2 and RBF 9-15-2 networks). The effectiveness of those networks is high and amounts to over $98.5 \%$, using the RBFT learning algorithm and the output softmax activation function. The result achieved by this network is less than $0.5 \%$ lower than the quality of the best MLP network using the same learning set (over $99 \%$ ). After analyzing various structures of the neural network using the polynomial coefficients with a degree of $\mathrm{N}=3$, it was empirically confirmed that the best learning results are achieved by a network of 19 neurons in a hidden layer (RBF network 12-19-2). The effectiveness of this network is almost $98 \%$. This result, as in the case of the RBF network using second-degree coefficients, is slightly lower than the effectiveness of the MLP network learned on the basis of the same data (this difference is less than $1 \%$ ).

Based on the conducted experiments, it was found that despite the common opinion in the literature [20] (pp. 31-38) [21] (pp. 210-216), that radial networks are much better suited than MLP networks for solving tasks in the category of solving patterns, the tested MLP networks achieved a slightly better efficiency, i.e., the learning error of those networks, was less than the learning error of the RBF networks.

For a thorough analysis of the error, the process of teaching the network with selected architectures (i.e., with the highest efficiency-networks using the second-degree coefficients with the architecture 9-22-2 and networks using the third-degree coefficients with the architecture 12-16-2) was checked in the Matlab environment. Due to the random selection of input neurons by the network and another network learning algorithm, the learning results (i.e., the quality of the network) may differ from those presented in Section 4. In addition, the Neural Network Toolbox package only allows the selection of a tool (i.e., a neural network) due to its purpose, apart from the choice of the type of network being tested. Therefore, a pattern recognition network was used by selecting the Pattern Recognition Tool. Figures 6 and 7 show a general structure diagram of both neural networks described using the Neural Network Pattern Recognition Tool from the Neural Network Toolbox package in Matlab environment. 


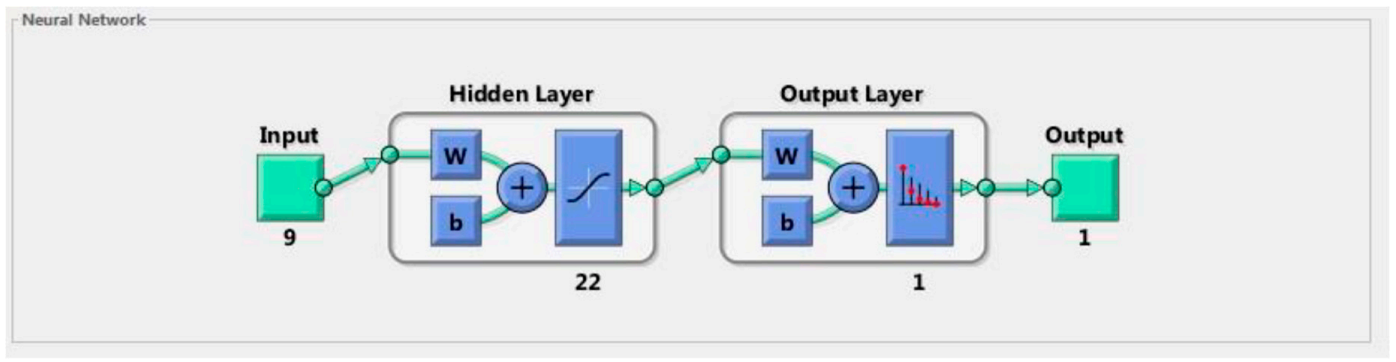

Figure 6. General structure of the applied neural network using polynomial coefficients with the degree $\mathrm{N}=2$.

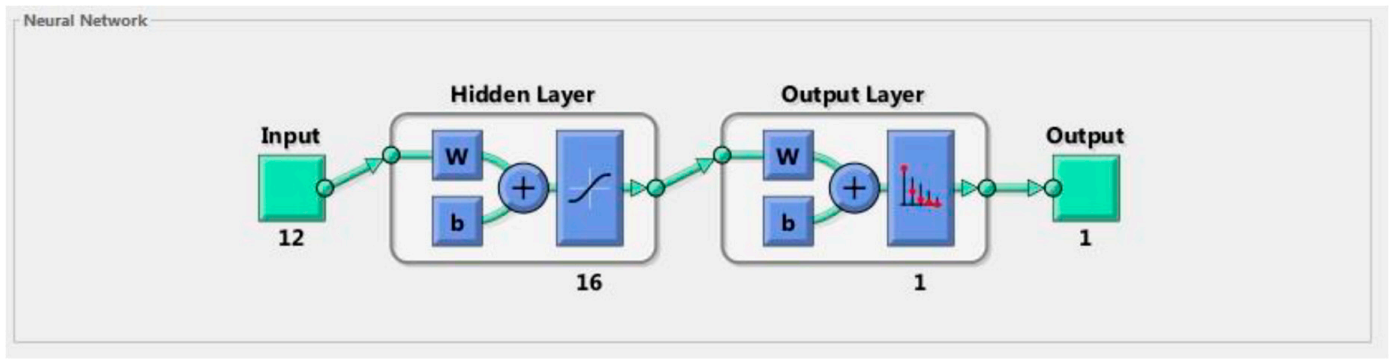

Figure 7. General structure of the applied using neural network polynomial coefficients with the degree $\mathrm{N}=3$.

During network teaching, the input data, according to the principles of neural network learning, were divided into three sets. The first set, learning set, included 459 packages (70\% of all data). The second set (validation set), used for validation and protection against the effects of network overlearning, included 99 packets (15\% of all data), similarly to the third set, used for testing the network, i.e., checking its ability to generalize (test set). The distribution of training data set was the same for both networks.

A maximum learning time of 1000 epochs was assumed for network learning. The epoch is the process of processing the entire learning sequence. This process is repeated until the epoch error falls below the allowable error. However, in reality, this time was much shorter and amounted to 104 epochs for the network using second degree coefficients and 12 epochs for the network using third degree coefficients. Figure 8 presents windows describing the learning process of one of the analyzed networks in the Matlab environment.

\section{Assessment of Network Efficiency and Error Behavior}

The effectiveness of the artificial neural network in the process of preset classification was assessed using indicators for the effectiveness assessment. Those indicators included: LQ learning quality, VQ validation quality, TQ testing quality, LE learning error, VE validation error, TE testing error and average error. Those indicators were read from the error matrix generated in Matlab (Figures 9 and 10). Errors from all three sets: learning, validation and test mean the value of incorrectly classifying of objects from one class to another. 


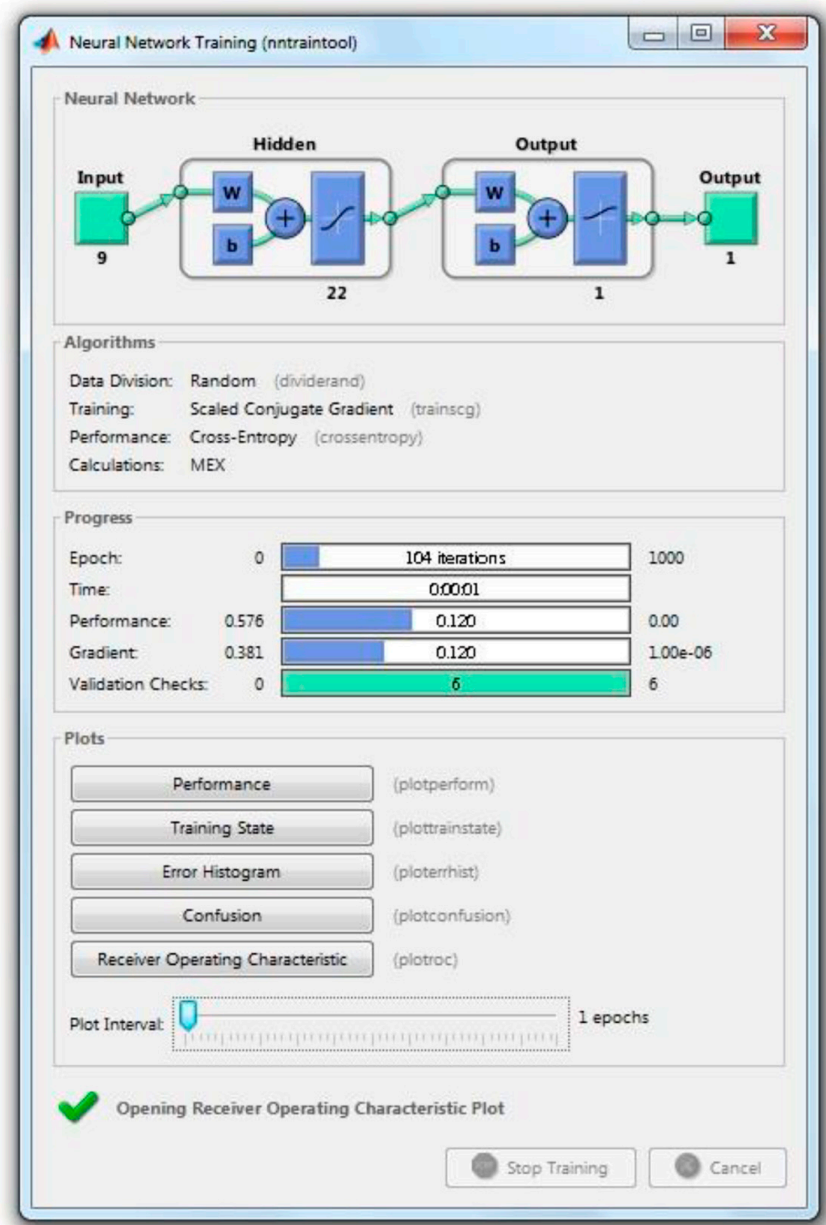

Figure 8. Process of learning of neural network using polynomial coefficients with the degree $\mathrm{N}=2$.
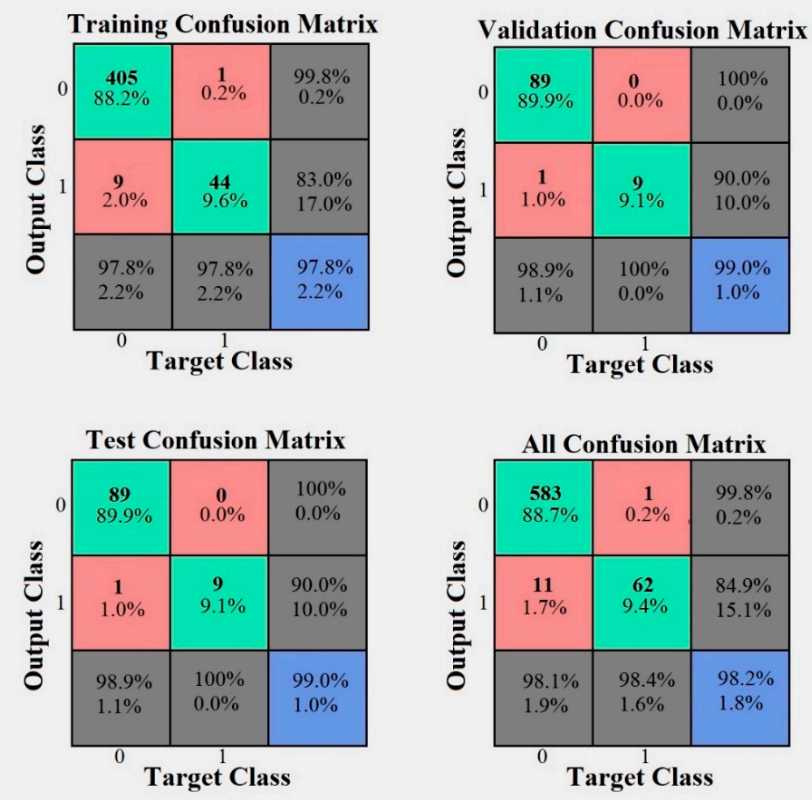

Figure 9. Error matrix of a network using polynomial coefficients with the degree $\mathrm{N}=2$. 

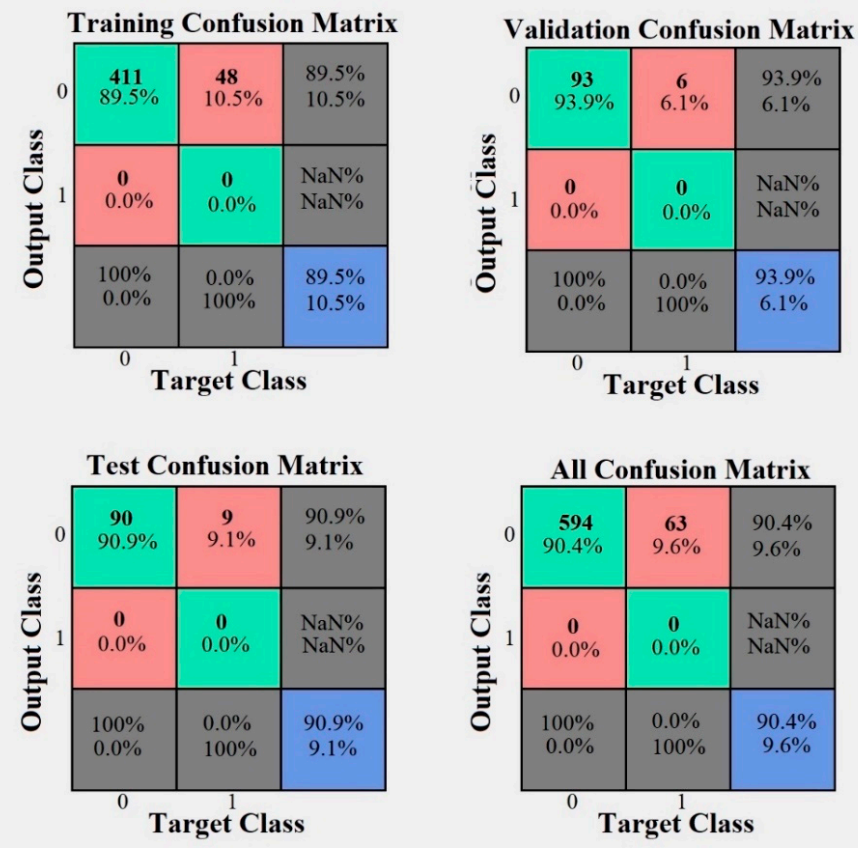

Figure 10. Error matrix of a network using polynomial coefficients with the degree $N=3$.

As can be seen in the figures, the average learning error for a network using the second degree polynomial coefficients did not exceed $2 \%$, and, for a network using the third degree polynomial coefficients, it was much higher and amounted to $9.6 \%$. The average network quality was respectively $98.2 \%$ for networks using second degree coefficients and $90.4 \%$ for networks using third degree polynomial coefficients.

The characteristics of error changes during the entire learning process of both neural networks are shown in Figures 11 and 12.

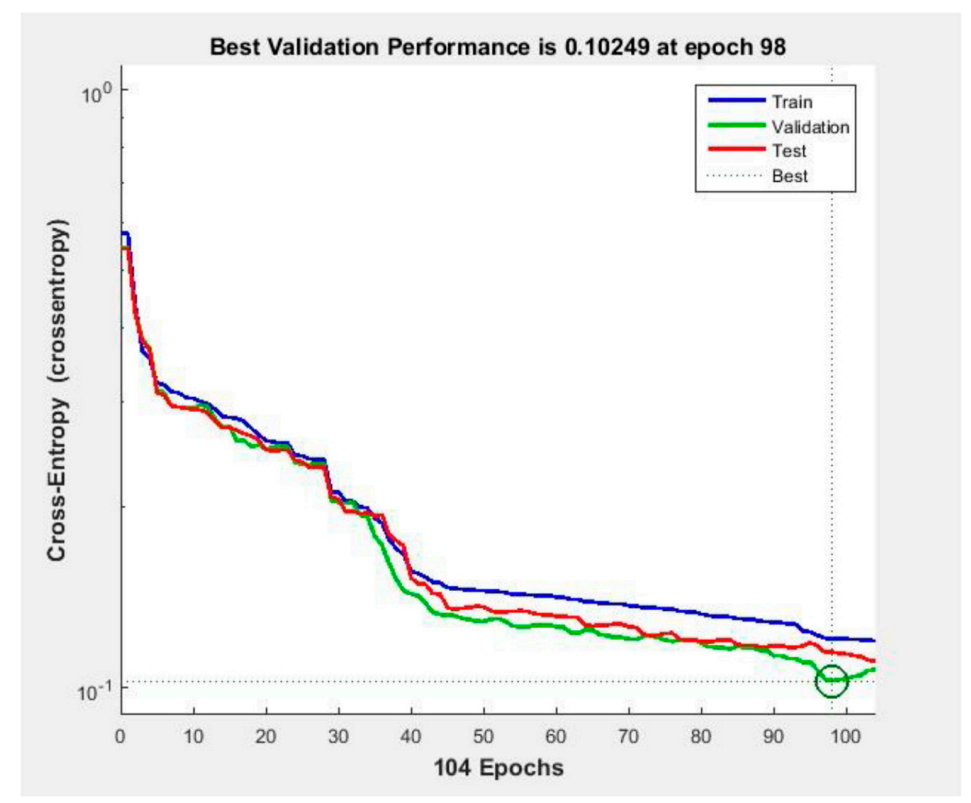

Figure 11. Characteristics of error changes during the learning process of a network using second-degree polynomial coefficients. 


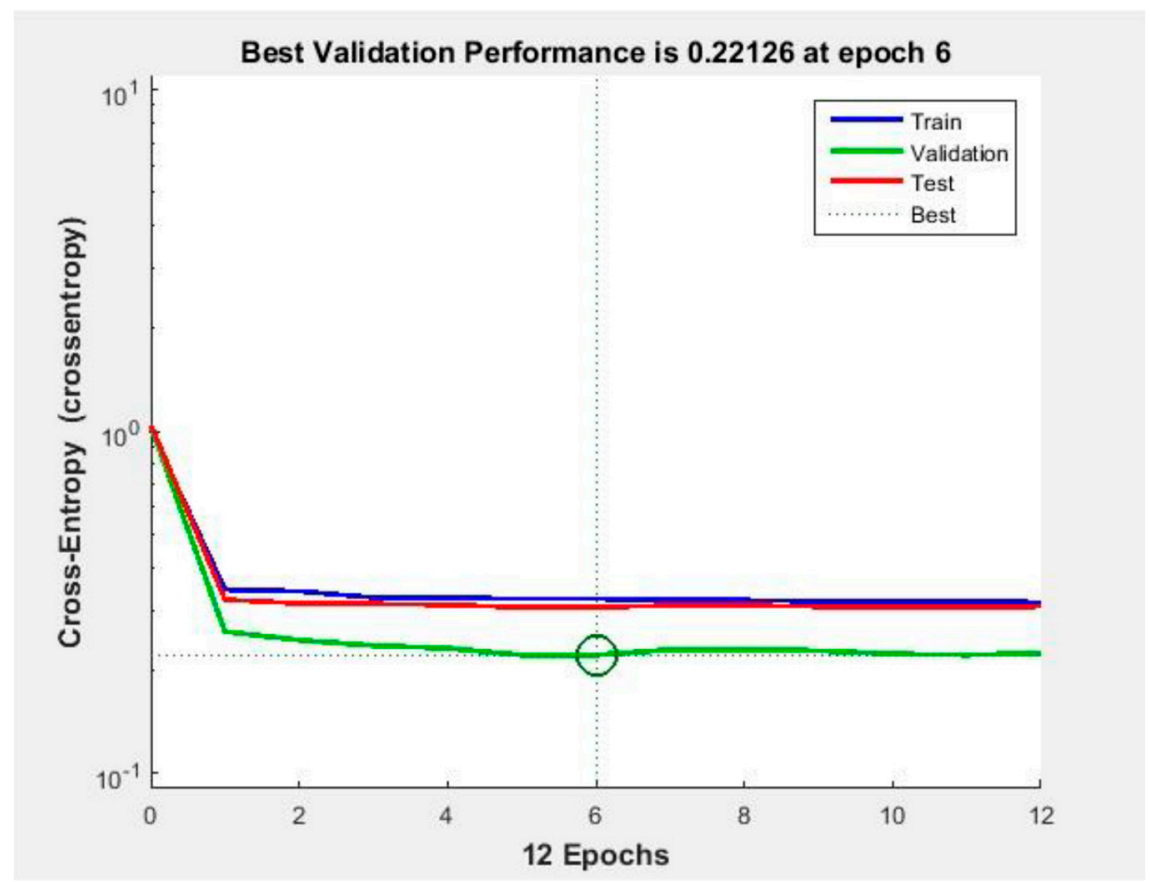

Figure 12. Characteristics of error changes during the learning process of a network using third-degree polynomial coefficients.

The moment when the error calculated for the validation set begins to increase (instead of decreasing with the decrease of the error for the learning set) signals the effect of overfitting the network [11]. The graphs show that for a network using second degree coefficients this moment occurred in the 98th epoch of the learning process (Figure 11), and for a network using the third degree coefficients-in the sixth epoch of the learning process (Figure 12).

The graphs in Figures 11 and 12 show the process of reducing the average error. In order to examine the errors made by the network for individual data, one should analyze the histograms of the error values presented for both networks in Figures 13 and 14, respectively.

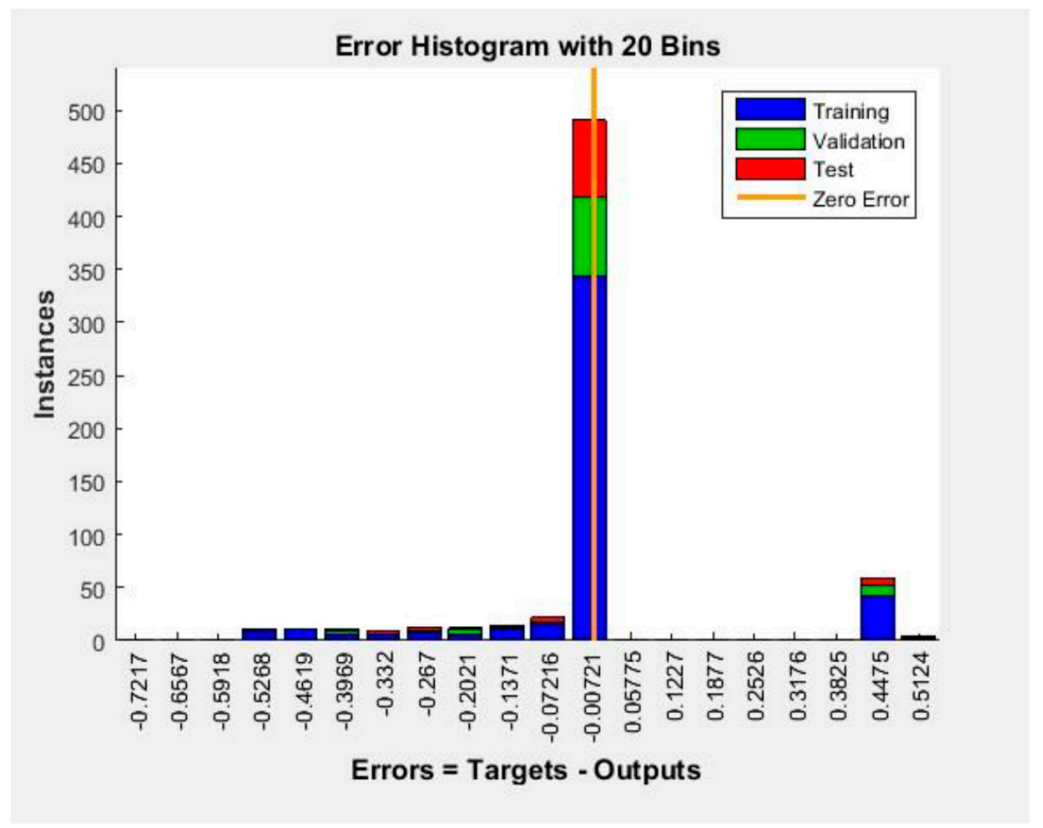

Figure 13. Histogram of errors made by the network using second-degree polynomial coefficients. 


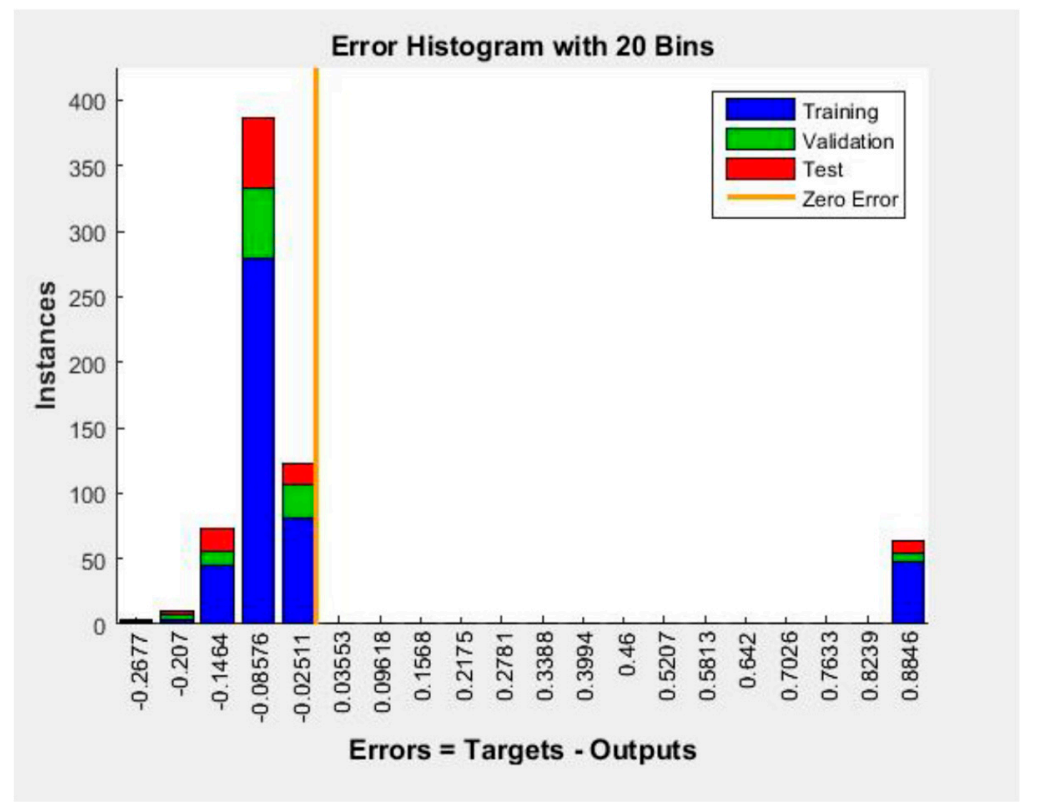

Figure 14. Histogram of errors made by the network using third-degree polynomial coefficients.

The assessment of the operation of the network using the second degree coefficients on the basis of the error histogram shows that the network recognizes correct and incorrect drive packets with a very small error. The vertical orange line on the histogram indicates a zero error. As can be seen in Figure 13, the largest bars of errors made by the network are located near zero. Larger error values were rare and occurred mostly in the training set [11] (pp. 955-960).

The histogram shown in Figure 14 shows the errors made by the network using third degree coefficients. As you can see in the histogram, the bars representing individual errors are larger than those in the histogram of Figure 13, and the bars of the largest errors are further away from zero. Although the network made the most errors in the learning set, and the error values are close to zero, it has a slightly lower efficiency than a network using second degree coefficients.

The value of the mean absolute error MAE (mean absolute error) was also calculated according to the formula:

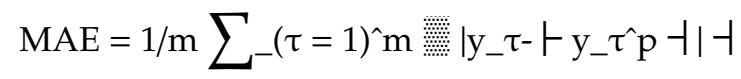

and the value of the mean RMSE error (root-mean square error) of prediction corresponding to the root mean square error, according to the formula:

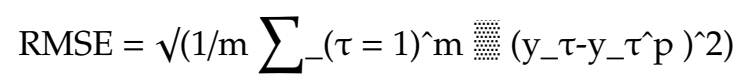

$\mathrm{y}_{\tau}$-actual value (result of tests),

$\mathrm{y}_{\tau}{ }^{\mathrm{p}}$-predicted value,

$\mathrm{m}$ - the number of predictions for the value of the variable.

The MAE and RMSE error values of selected neural networks were analyzed. The values of the prediction error and the average RMSE prediction error for the selected network using polynomial coefficients with the degree $\mathrm{N}=2$ and network using polynomial coefficients with the degree $\mathrm{N}=3$ are presented in the Table 4 below. The number of predictions for the value of the variable was 57 .

The task set for both neural networks was to recognize by those networks the moment of sufficient operator training. In practice, this moment meant the interruption of training (the condition for stopping the training was triple, error-free driving a preset section). 
Table 4. MAE and RMSE error values of selected neural networks.

\begin{tabular}{llc}
\hline & MAE & RMSE \\
\hline Selected neural network using polynomial coefficients with the degree $\mathrm{N}=2$ & 0.052632 & 0.229416 \\
Selected neural network using polynomial coefficients with the degree $\mathrm{N}=3$ & 0.087719 & 0.296174 \\
\hline
\end{tabular}

Both networks learned to recognize the operator's level of learning are very likely to indicate when he acquired the right skills. However, to solve the problem posed in the article, a network using second-degree coefficients should be used, because this network is characterized by the highest quality in all: teaching, test and validation sets.

\section{Discussion}

The scope of the research included the issues of training the operator of selected devices used in precision agriculture. The main research problem was the analysis of the possibility of using an artificial neural network to assess the degree of training of the operator of a vehicle driven according to the indications of the navigation panel on parallel lanes. A neural model was used to prove the research goal. The task of the analyzed neural network was to assign information on sufficient or insufficient training of the operator driving the agricultural tractor in accordance with the navigation panel indications. The use of artificial intelligence methods in agriculture is wide-ranging, but so far these methods have not been used to assess the degree of training of operators. Their level of training was judged arbitrarily by observation. For this reason, the training time was often too long, which generated, for example, costs related to the fuel needed to perform test drives.

The presented results confirm the effectiveness of the applied drive classification process based on the method using an artificial neural network. The simulation results clearly confirm the hypothesis formulated, assuming that the moment of training operators can be automatically recognized by a properly selected artificial neural network, learned on the basis of data collected during the observation of the operator training process, using a criterion specified by experts.

\section{Conclusions}

The following conclusions emerge from the tests and analyzes conducted:

1. Artificial neural networks can automatically recognize the moment of sufficient training of an operator driving a vehicle according to the indications of navigation on parallel strips.

2. This network can be learned on the basis of empirical data collected during the observation of the operator training process under the control of experienced instructors who assess the level of training according to the adopted assessment criterion.

3. An artificial neural network implemented in the driver training monitoring device will allow tracking of training progress and signaling the achievement of the required level of training (in some cases this may mean a shortening of the training cycle, and sometimes it may mean that, despite the completion of the assumed number of exercises, the operator still does not have the required level of skill).

4. A number of drivers acquire the desired tractor steering skills faster than the training program requires. Identification of such drivers allows to reduce training costs (as they do not have to continue training until the end of the planned program).

5. Replacing the supervision of a real trainer with the assessment of drives made automatically by means of an artificial neural network will allow the subjective assessment to be replaced by an objective assessment generated by an electronic device, which may increase the effectiveness of training.

6. A review of the literature made by the authors permits the statement that the solution to the scientific problem has features of originality, because the method proposed in the article using an 
artificial neural network has not yet been used to solve the problem of assessing the degree of operator training.

As mentioned in the introduction, many scientists conduct research on the automatic driving of a vehicle along designated paths $[9,10]$. Farmers who use precision driving navigation often cannot benefit from the knowledge of an expert to objectively assess their skills, as was the case with the research training for this article. Implementation of a system based on the operation of artificial neural networks, which will enable automatic recognition of the operator's training, not only provide him with confidence in operation, but also shorten the time of possible training or self-training.

Author Contributions: Conceptualization, K.T. and A.K.; methodology, K.T. and A.K.; formal analysis, K.T.; writing-preparing an original project, K.T. and A.K.; writing-reviewing and editing, K.T. and A.K. All authors have read and agreed to the published version of the manuscript.

Funding: This research received no external funding.

Conflicts of Interest: The authors declare no conflict of interest.

\section{References}

1. Trzyniec, K. Sposoby badania obciążenia psychicznego pracą. In Dokonania Naukowe Doktorantów-Creative Science; Kuczera, M., Ed.; Creativetime Time: Poland, Kraków, 2013; Volume 1, pp. 138-139.

2. Trzyniec, K.; Juliszewski, T.; Kowalewski, A. Assessment of the degree of training of the operator of state-ofthe-art signalling and control devices. Tech. Rol. Ogrod. Leśna 2015, 2, 12-15.

3. Trzyniec, K.; Kowalewski, A. Symbole kodowania informacji na urządzeniach sygnalizacyjnych i sterowniczych ciągników i maszyn rolniczych. J. Res. Appl. Agric. Eng. 2016, 2, 120-122.

4. Inoue, K.; Kaizu, Y.; Igarashi, S.; Imou, K. The development of autonomous navigation and obstacle avoidance for a robotic mower using machine vision technique. IFAC-PapersOnLine 2019, 52, 173-177. [CrossRef]

5. Gonzalez-de-Santos, P.; Fernandez, R.; Sepúlveda, D.; Navas, E.; Armada, M. Unmanned Ground Vehicles for Smart Farms. In Agronomy_Climate Change \& Food Security; Amanullah, K., Ed.; IntechOpen: London, UK, 2020; pp. 388-392.

6. Chemhengcharoen, P.; Nilsumrit, P.; Pongpetrarat, P.; Phanomchoeng, G. Development of a Prototype of Autonomous Vehicle for Agriculture Applications. In Proceedings of the 7th International Conference on Communications and Broadband Networking, Nagoya, Japan, 12-15 April 2019; Association for Computing Machinery: New York, NY, USA, 2019; pp. 53-57.

7. Hasníková, M.; Staniszewska, E.; Lisec, A. Possible Use of Autonomous Vehicles in Agriculture, XII. In Proceedings of the International Conference on Logistics in Agriculture, Novo Mesto, Slovenia, 15 November 2018.

8. Petterson, T.C. Development of an Electric Vehicle for Autonomous Use on a New Zealand Dairy Farm. Master's Thesis, Mechanical Engineering University of Canterbury, Christchurch, New Zealand, 2020.

9. Samuel, M.; Mohamad, M.; Hussien, M.; Godi, N. Control of Autonomous Vehicle Using Path Tracking: A Review. J. Comput. Theor. Nanosci. 2018, 24, 3877-3879. [CrossRef]

10. Ashraf, M.A.; Takeda, J.; Torisu, R. Neural Network Based Steering Controller for Vehicle Navigation on Sloping Land. Eng. Agric. Environ. Food 2010, 3, 100-104. [CrossRef]

11. Szymczyk, P.; Tomecka-Suchoń, S.; Szymczyk, M. Supervised and unsupervised learning in radial basis function classifers. Int. J. Appl. Math. 2015, 25, 955-960.

12. Stanisz, A. Przystępny Kurs Statystyki z Zastosowaniem STATISTICA PL na Przykładach z Medycyny, 1st ed.; StatSoft Polska: Krakow, Poland, 2006.

13. Lewandowski, S. Modelowanie i Klasyfikacja Połaczeń Końców Przędz Przy użyciu Sztucznych Sieci Neuronowych, 1st ed.; Wydawnictwo Akademii Techniczno-Humanistycznej: Bielsko-Biała, Poland, 2009.

14. STATISTICA; Neural NetworksTM PL. Kurs Użytkownika Programu na Przykładach; Statsoft: Krakow, Poland, 2001.

15. STATISTICA; Neural NetworksTM PL. Poradnik Użytkownika; Statsoft: Krakow, Poland, 2001.

16. STATISTICA; Neural NetworksTM PL. Przewodnik Problemowy; Statsoft: Krakow, Poland, 2001.

17. STATISTICA; Neural NetworksTM PL. Wprowadzenie do Sztucznych Sieci Neuronowych; Statsoft: Krakow, Poland, 2001. 
18. Kacprzyk, J. Foreword. In Multilayer Neural Systems and Generalized Net Models; Akademicka Oficyna Wydawnicza EXIT: Warszawa, Poland, 2009.

19. Nabney, I. Netlab: Algorithms for Pattern Recognition. Advances in Pattern Recognition; Springer: Birmingham, UK, 2001.

20. Leonard, J.A.; Kramer, M.A. Radial basis function networks for classifying process faults. IEEE Control Syst. Mag. 1991, 11, 31-38.

21. Tarassenko, L.; Roberts, S. Supervised and unsupervised learning in radial basis function classifers. IEEE Proc. Vis. Image Signal Process. 1994, 141, 210-216. [CrossRef]

Publisher's Note: MDPI stays neutral with regard to jurisdictional claims in published maps and institutional affiliations.

(C) 2020 by the authors. Licensee MDPI, Basel, Switzerland. This article is an open access article distributed under the terms and conditions of the Creative Commons Attribution (CC BY) license (http://creativecommons.org/licenses/by/4.0/). 